ISSN: 0210-7287

DOI: http://dx.doi.org/10.14201/161620199263272

\title{
TRADUCCIÓN INDIRECTA Y DIRECTA DEL CHINO AL ESPAÑOL: LA OBRA DE MO YAN EN ESPAÑA
}

\author{
Indirect and Direct Translation from Chinese \\ to Spanish: The Work of Mo Yan
}

\author{
Yu Jingxian \\ Universidad de Santiago de Compostela \\ yujingxian94@botmail.com
}

Recibido: junio de 2019; Aceptado: septiembre de 2019; Publicado: diciembre de 2019

Ref. Bibl. YU JINGXIAN. TRADUCCIÓN INDIRECTA Y DIRECTA DEL CHINO

AL ESPAÑOL: LA OBRA DE MO YAN EN ESPAÑA. 1616: Anuario de Literatura

Comparada, 9 (2019), 263-272

RESUMEN: La literatura china ocupa una parte muy pequeña de las traducciones publicadas en España; además, a lo largo del flujo de la difusión de literatura china se destaca el fenómeno de traducción indirecta del chino al español a partir de versiones, principalmente en inglés. Sin embargo, con la concesión del Premio Nobel de Literatura en 2012 al escritor chino Mo Yan, se traducen directamente más obras de él y otros escritores chinos. Este trabajo se centra en presentar el fenómeno de traducción indirecta y directa del chino tomando como ejemplos La vida y la muerte me están desgastando y El suplicio del aroma de sándalo de Mo Yan. También se mostrará el cambio de fuente de traducción en términos de traductores al español y la editorial con la responsabilidad principal en la traducción de obras de Mo Yan al español. Yan.

Palabras clave: traducción indirecta; traducción directa; chino; español; Mo 
ABSTRACT: Chinese literature occupies a very small part of the translations published in Spain; in addition, along the flow of the diffusion of Chinese literature stands out the phenomenon of indirect translation from Chinese to Spanish from versions, mainly, in English. However, with the award of the Nobel Prize for Literature to the Chinese writer Mo Yan in 2012, more works by him and other Chinese writers are directly translated. This work will focus on presenting the phenomenon of indirect and direct translation of Chinese, taking as examples Life and Death Are Wearing Me Out and Sandalwood Death by Mo Yan. In addition, the change of translation source will also be showed in terms of Spanish translators and press of Mo Yan's works.

Key words: Indirect Translation; Direct Translation; Chinese; Spanish; Mo Yan.

Según los datos de Statista de 2017, en España, entre 2008 y 2017 se publicaron alrededor de 20.772 traducciones por año como promedio, que representan más del $20 \%$ de sus publicaciones. Entre las obras traducidas, el $51 \%$ tienen el inglés como lengua fuente, el $10 \%$ el italiano y el $9,5 \%$ el francés. El japonés es la lengua asiática que se traduce más, con un 3\%, mientras que el chino solo representa un $0,1 \%{ }^{1}$. Este pequeño porcentaje no solo se debe a la poca difusión de la literatura china, sino también a la gran cantidad de traducciones indirectas de obras escritas originalmente en chino, que fueron traducidas al español a través de versiones inglesas o de otros idiomas europeos, como el francés. En este artículo, tomo como caso concreto la traducción al español de obras de Mo Yan, el ganador del Premio Nobel de Literatura 2012, para presentar el fenómeno de traducción indirecta y directa del chino al español.

\section{LAS TRADUCCIONES DE OBRAS DE MO YAN}

Hasta junio de 2019, en España se han publicado en total diecisiete títulos de Mo Yan. Todos se tradujeron al castellano, además de uno al catalán y uno al euskera. En la Tabla 1 se recogen las primeras ediciones españolas de cada obra.

1. Los datos provienen de Statista: <https://es.statista.com/estadisticas/473695/ libros-traducidos-por-idioma-original-espana/> (consulta 26.06.2019). 
YU JINGXIAN

TRADUCCIÓN INDIRECTA Y DIRECTA DEL CHINO AL ESPAÑOL: LA OBRA DE MO YAN EN ESPAÑA

\begin{tabular}{|c|c|c|c|c|c|}
\hline Año & Título & $\begin{array}{l}\text { Lengua } \\
\text { original }\end{array}$ & $\begin{array}{l}\text { Lengua } \\
\text { meta }\end{array}$ & Traductores & Editorial \\
\hline 1992 & $\begin{array}{l}\text { Sorgo rojo } \\
\text { (红高粱) }\end{array}$ & inglés & castellano & Ana Pojak & $\begin{array}{l}\text { El Aleph } \\
\text { (Muchnik) }\end{array}$ \\
\hline 2007 & $\begin{array}{l}\text { Grandes pechos, am- } \\
\text { plias caderas } \\
\text { (丰乳肥臀) }\end{array}$ & inglés & castellano & $\begin{array}{l}\text { Mariano } \\
\text { Peyrou }\end{array}$ & Kailas \\
\hline 2008 & $\begin{array}{l}\text { Las baladas del ajo } \\
\text { (天堂蒜臺之歌) }\end{array}$ & inglés & castellano & Carlos Ossé & Kailas \\
\hline 2009 & $\begin{array}{l}\text { La vida y la muerte } \\
\text { me están desgastando } \\
\text { (生死疲劳) }\end{array}$ & inglés & castellano & Carlos Ossé & Kailas \\
\hline 2010 & $\begin{array}{l}\text { La república del vino } \\
\text { (酒国) }\end{array}$ & inglés & castellano & Cora Tiedra & Kailas \\
\hline 2011 & $\begin{array}{l}\text { Shifu, harias cual- } \\
\text { quier cosa por } \\
\text { divertirte } \\
\text { (师傅愈来愈幽默) }\end{array}$ & inglés & castellano & Cora Tiedra & Kailas \\
\hline 2011 & Rana (蛙) & chino & castellano & Li Yifan & Kailas \\
\hline 2012 & Cambios (变) & chino & castellano & $\begin{array}{l}\text { Anne-Hélène } \\
\text { Suárez } \\
\text { Girard }\end{array}$ & Seix Barral \\
\hline 2012 & Canvis (变) & inglés & catalán & $\begin{array}{l}\text { Carles Prado } \\
\text { Fonts }\end{array}$ & Edicions 62 \\
\hline 2013 & $\begin{array}{l}\text { Hori da umbrae, } \\
\text { maisu } \\
\text { (师傅愈来愈幽默) }\end{array}$ & inglés & euskera & $\begin{array}{l}\text { Maialen Ma- } \\
\text { rin/ Aiora } \\
\text { Jaka }\end{array}$ & Elkar \\
\hline 2013 & $\begin{array}{l}\text { ¡Boom! } \\
\text { (四十一炮) }\end{array}$ & chino & castellano & Li Yifan & Kailas \\
\hline 2014 & $\begin{array}{l}\text { El suplicio del aroma } \\
\text { de sándalo } \\
\text { (檀香刑) }\end{array}$ & chino & castellano & $\begin{array}{l}\text { Blas Piñero } \\
\text { Martínez }\end{array}$ & Kailas \\
\hline 2015 & $\begin{array}{l}\text { Trece pasos } \\
\text { (十三步) }\end{array}$ & chino & castellano & $\begin{array}{l}\text { Juan José Ci- } \\
\text { ruela Alférez }\end{array}$ & Kailas \\
\hline 2016 & $\begin{array}{l}\text { El manglar } \\
\text { (红树林) }\end{array}$ & chino & castellano & $\begin{array}{l}\text { Blas Piñero } \\
\text { Martínez }\end{array}$ & Kailas \\
\hline
\end{tabular}


TRADUCCIÓN INDIRECTA Y DIRECTA DEL CHINO AL ESPAÑOL: LA OBRA DE MO YAN EN ESPAÑA

\begin{tabular}{|c|c|c|c|c|c|}
\hline Año & Título & $\begin{array}{l}\text { Lengua } \\
\text { original }\end{array}$ & $\begin{array}{l}\text { Lengua } \\
\text { meta }\end{array}$ & Traductores & Editorial \\
\hline 2016 & $\begin{array}{l}\text { El clan del sorgo rojo } \\
\text { (红高粱家族) }\end{array}$ & chino & castellano & $\begin{array}{l}\text { Blas Piñero } \\
\text { Martínez }\end{array}$ & Kailas \\
\hline 2017 & $\begin{array}{l}\text { El mapa del tesoro } \\
\text { escondido (藏宝图) }\end{array}$ & chino & castellano & $\begin{array}{l}\text { Blas Piñero } \\
\text { Martínez }\end{array}$ & Kailas \\
\hline 2017 & $\begin{array}{l}\text { El rábano transparente } \\
\text { (透明的红夢卜) }\end{array}$ & chino & castellano & $\begin{array}{l}\text { Blas Piñero } \\
\text { Martínez }\end{array}$ & Kailas \\
\hline 2018 & $\begin{array}{l}\text { El clan de los } \\
\text { herbivoros } \\
\text { (食草家族) }\end{array}$ & chino & castellano & $\begin{array}{l}\text { Blas Piñero } \\
\text { Martínez }\end{array}$ & Kailas \\
\hline 2019 & $\begin{array}{l}\text { Una carrera en obras } \\
\text { (筑路) }\end{array}$ & chino & castellano & $\begin{array}{l}\text { Blas Piñero } \\
\text { Martínez }\end{array}$ & Kailas \\
\hline
\end{tabular}

Tabla 1: Obras de Mo Yan publicadas en España (1992-2019).

Fuente: Base de datos de libros editados en España y Kailas (elaboración propia).

Desde la primera traducción en 1992 hasta 2011, en España se publicaron seis títulos de Mo Yan. Todas las traducciones se realizaron a partir de las versiones en inglés de Howard Goldblatt ${ }^{2}$. A través de los títulos, se detecta ya la relación entre las versiones de ambos idiomas, cuyas formas y palabras manifiestan una notable correspondencia. Por ejemplo, «红高粱》 (literalmente, 'sorgo rojo') se traduce en inglés como Red Sorghum y en español Sorgo Rojo, y «丰乳肥臂” (literalmente, 'pechos y glúteos gordos') se traduce al inglés como Big Breasts and Wide Hips y al español como Grandes pechos, amplias caderas. Como las fuentes de las traducciones españolas son los textos en inglés de Goldblatt, que se traducen a veces de forma literal, a veces mediante paráfrasis y otras incluso con omisión de pasajes difíciles con el objeto de hacer las traducciones fluidas y comprensibles para los lectores (Meng 2014), los resultados de las traducciones del inglés al español presentan importantes diferencias en relación con los textos originales chinos.

Uno de los problemas más destacados es la traducción errónea. En el caso de La vida y la muerte me están desgastando. Como en su mayor parte la traducción española es muy fiel a la versión intermediaria en inglés, cuando se dan errores en la inglesa pasan a la española. Por ejemplo, la

2. Este dato proviene de "China traducida y por traducir», <http://china- traducida. net/autores/mo-yan/> (consultado 29.07.2019). 
palabra «冬瓜» (Benincasa hispida, calabaza blanca) (Mo 2006, 37) se traduce en inglés por gourd (Goldblatt 2008, 23) y en español por "calabaza" (Ossés 2009 , 41). A pesar de que la calabaza blanca y la calabaza pertenecen a la misma familia, no son iguales ni en la forma ni en el color. Aparte de los errores comunes en ambas traducciones, el traductor español también cometió algunos errores que no están presentes en la versión inglesa. En el capítulo 3, «杏树》 (albaricoquero) (Mo 2006, 35), por ejemplo, se traduce en inglés «the apricot tree" (Goldblatt 2008, 21), pero en español se opta por "el melocotonero" (Ossés 2009, 30), aunque es evidente que no se trata del mismo árbol. Además, en el capítulo 33 se proporciona la forma correcta, de modo que es muy probable que el primer caso se deba a un descuido del traductor. Otro ejemplo es «毛主席语录》, un libro que recoge las citas de Mao Zedong y se traduce al inglés Little Red Book (Goldblatt 2008, 157), título que el traductor español calca como Pequeño libro rojo (Ossés 2009, 228), cuando se lo conoce en realidad como Libro rojo de Mao. Posiblemente la solución adoptada se debe a que el traductor desconoce este libro o traduce descuidadamente, con lo que los lectores fácilmente se confundirán o no llegarán a identificar el significado real (Ku 2010).

Además de la traducción errónea, otro problema notable tiene que ver con los contenidos suprimidos. A consecuencia de que Goldblatt suprimió algunos contenidos en el proceso de la traducción, es imposible que aparezcan en el texto español. Esto ocurre especialmente en las frases hechas, tales como "Chengyu ${ }^{3}$, adagios y expresiones con historia que no son conocidas por los extranjeros. Véase al respecto este ejemplo del capítulo 19:

\footnotetext{
但一个二十多岁就当了县级领导干部的人, 和农村姑娘结婚的可能性为 零，无论她貌如西施还是色比婵娟。(Mo 2006, 197)

[No había la menor posibilidad de que un importante líder a nivel del condado, de veinte años, llegara realmente a casarse con una campesina, sea parecida a Xi Shi o sea comparable a Chan Juan] (mi traducción; el énfasis es mío).

There was no chance that a leading county-level cadre in his twenties would ever actually marry a peasant girl, no matter how pretty or fetching she might be (Goldblatt 2008, 198; el énfasis es mío).

No había la menor posibilidad de que un importante líder a nivel del condado, de veinte años, llegara realmente a casarse con una campesina, por muy hermosa o atractiva que fuera (Ossés 2009, 288; el énfasis es mío).
}

3. Se trata de una expresión idiomática característica del idioma chino, que suele consistir en cuatro caracteres y tiene su origen en la literatura antigua. 
En la cultura china, 西施 (Xi Shi) y 婵娟 (Chan Juan) representan desde la antigüedad dos figuras femeninas caracterizadas por su belleza y suelen usarse para describir a una mujer muy atractiva. Sin embargo, estas expresiones metafóricas no son conocidas por los extranjeros, por lo que fueron eliminadas para evitar los obstáculos. De hecho, esto supone una especie de amputación del estilo de Mo Yan, que se caracteriza por el uso de este tipo de expresiones.

Mediante estos ejemplos de la traducción de La vida y la muerte me están desgastando pretendo demostrar que, aunque el texto en inglés ofrecía una alternativa más fácil cuando la traducción chino-española todavía no estaba madura en España, la traducción indirecta es una opción limitada con respecto al original, que aleja a los lectores del texto original y la cultura china. Además de la traducción propiamente dicha, la dependencia del sistema literario anglófono también influye en la selección y la difusión de las obras. Los editores primero leen los libros en inglés, que son propuestos por agentes literarios, y después toman las decisiones. En lo que se refiere a la publicación, este proceso puede verse reflejado en una entrevista a Mo Yan de 2007: «Yo envié el cuestionario en castellano e inglés, y un intérprete lo tradujo al chino. Las respuestas de Mo Yan fueron traducidas del chino al inglés y posteriormente al castellano" (en Marín 2012, 402).

Otro fenómeno agrava el alejamiento, a saber, la traducción camuflada. En las páginas de crédito de las seis obras traducidas a partir del inglés, solo las últimas dos (La república del vino y Shifu, haría cualquier cosa por divertirte) mencionan el nombre del traductor Howard Goldblatt y los títulos en inglés, mientras que las primeras cuatro son traducciones indirectas y camufladas en la medida en que no mencionan el nombre del traductor de chino a inglés, como si hubieran sido traducidas directamente de chino a español. Este fenómeno de traducción camuflada no solo ocurre en el caso de Mo Yan, sino también en muchas otras obras de literatura china a partir de traducciones a inglés o francés.

La primera traducción directa de obras de Mo Yan tuvo lugar en 2011 cuando se publicó Rana en traducción de Li Yifan. Después de este libro, todas las traducciones posteriores se realizaron directamente del chino al español, excepto dos traducciones a otras lenguas españolas, Canvis (2012) en catalán y Hori da umorea, maisu! (2013) en euskera, que traducen del inglés. Este cambio de la traducción indirecta a la directa se debe, principalmente, al aumento de la cantidad de los traductores chino-españoles a partir del siglo xxi. Además, el Premio Nobel también es un factor crucial, gracias al cual la editorial se atreve a invertir en las traducciones directas del chino.

Para comparar las traducciones indirectas y directas, tomo como muestras los primeros párrafos de El suplicio del aroma de sándalo como representante 
de la traducción directa y La vida y la muerte me están desgastando como representante de la traducción indirecta. En el primer párrafo de El suplicio del aroma de sándalo se registran 1.568 palabras españolas, mientras el párrafo correspondiente del texto original en chino contiene 259 caracteres chinos, una proporción de 6 a 1 por tanto de la última. En cuanto a La vida y la muerte me están desgastando, los primeros párrafos contienen respectivamente 284 caracteres chinos y 297 palabras españolas, en práctica equivalencia. La traducción directa del chino al español traslada un contenido más abundante y completo que la indirecta. Como ya he mencionado, la traducción de $L a$ vida y la muerte me están desgastando es de segunda mano, por lo cual el fenómeno de omisión es muy frecuente. Además, la desproporción de unidades léxicas también tiene que ver con las diferentes soluciones traductológicas para objetos o frases hechas que no existen en el idioma meta.

En el primer párrafo de La vida y la muerte me están desgastando se traduce el lugar «阴曹地府 (yin cao di fu)» por “infierno». Sin embargo, en la cultura tradicional china, el "yin cao di fu» es el lugar donde están las almas de todos los muertos, mientras que el infierno solo es la parte donde se encarcela a los pecadores. Al igual que en este ejemplo, en la traducción indirecta hay muchos elementos representados con algunas palabras que contienen significados similares y existentes en la lengua meta, pero no exactamente iguales que los chinos.

Al contrario, en la traducción directa de El suplicio del aroma de sándalo, los elementos orientales y la características del texto original son objeto de un tratamiento más serio y prudente. El traductor incluye una «Nota sobre la traducción» en la que discute las dificultades de la traducción y su metodología. En cuanto a la traducción del texto, el traductor recurre a métodos de traducción fonética con pinyin ${ }^{4}$. Por ejemplo, la palabra «亲爹” se traduce como "qindie", pero con una nota explicativa. Con ello, por un lado, no se rompe la fluidez de la lectura y, por otro, los lectores pueden conocer más sobre la cultura y la historia chinas, según su interés. Como es obvio, la traducción directa no es perfecta, pues también contiene algunos errores. Este es el caso de “慈禧老佛爷》 (Cixi lao fo ye), la emperatriz viuda Cixi, en el primer capítulo, que se traduce como «el gran Buda clemente y misericordioso».

En comparación con las traducciones tempranas, las traducciones directas disminuyen la distancia entre los dos sistemas literarios y la literatura china es recibida por los lectores hispanohablantes de forma más completa

4. Se trata de un sistema de transcripción fonética del chino mandarín, que sustituyte los caracteres tradicionales chinos por una transcripción fonética. 
y exacta. Sin embargo, el desarrollo de la traducción desde chino todavía no ha alcanzado una madurez suficiente en España.

\section{LOS TRADUCTORES AL ESPAÑOL DE OBRAS DE MO YAN}

Las traducciones españolas de obras de Mo Yan fueron realizadas por once traductores diferentes: Ana Poljak, Mariano Peyrou, Carlos Ossés, Cora Tiedra, Carles Prado Fonts, Maialen Marin y Aiora Jaka (traductores de inglés a español) y Yifan Li, Anne-Hélène Suárez Girard, Juan José Ciruela Alférez y Blas Piñero Martínez (traductores de chino a español). Entre los primeros, mientras Ana Poljak y Carlos Ossés tenían más experiencia en la traducción del inglés principalmente (en el caso de Poljak, también del italiano) antes de encargarse de obras de Mo Yan, otros solo habían traducido pocas obras desde el inglés antes. En cuanto a los traductores desde el chino, se pueden dividir en dos clases. La primera incluye al traductor Yifan $\mathrm{Li}$, un nativo chino que traduce Rana (2011) y iBoom! (2013). Debido a su inexperiencia inicial como traductor literario, Kailas encargó a Cora Tiedra el papel de editor para examinar sus traducciones (Zhou 2015). Yifan Li domina los idiomas chino y español y, con la revisión del editor español, el texto resultante en español resulta fluido y comprensible. Pero, desde un punto de vista traductológico, todavía es necesario mejorar la técnica de traducción con el fin de reflejar tanto la trama como las expresiones del texto original. La segunda clase incluye a sinólogos españoles que no solo son especialistas en el idioma chino, sino que también cuentan con experiencia en la traducción de literatura china. A esta categoría pertenecen Anne-Hélène Suárez Girard, Blas Piñero Martínez y Juan José Ciruela Alférez, quienes se encargaron de las traducciones de obras de Mo Yan a partir del año 2012. Suárez Girard es profesora de la Universidad Autónoma de Barcelona y autora del manual Lengua china para traductores, además de traductora de obras clásicas, como Daodejing, y novelas contemporáneas, como ;Vivir!, de Yu Hua. Juan José Ciruela Alférez es profesor de chino en la Universidad de Granada, además de editor de las revistas El chino de hoy, El pensamiento lingüústico en la China clásica y Aprender y enseñar chino; había traducido Los viajes del buen Doctor Can antes del encargo de la obra de Mo Yan. Blas Piñero Martínez, quien destaca por su mayor cantidad de traducciones de obras de Mo Yan, cuenta con siete títulos hasta 2019; antes de encargarse de estos trabajos, había traducido la novela El camello Xiangzi, de Lao Tse, y una antología de poesía china moderna entre 1918 y 1949.

Desde la traducción chino-inglés-española hasta la traducción directa chino-española, desde traductores chinos hasta traductores españoles, 
desde traductores del inglés hasta sinólogos, la especialización del grupo de traductores refleja una mejor formación en la traducción desde el chino y una mayor demanda de literatura china en España a partir de estos últimos años.

\section{EDITORIALES QUE PUBLICAN OBRAS DE MO YAN}

Las obras de Mo Yan en España han sido promovidas, a lo largo del tiempo, por cinco editoriales. Edicions 62 y Elkar argitaletxea, que se centran en las literaturas en catalán y euskera, respectivamente, publicaron dos traducciones de obras de Mo Yan a estas dos lenguas. El Aleph (Muchnik) y Seix Barral se centran en narrativa (también ensayo y poesía en el caso de Seix Barral) en lengua española. Finalmente, Kailas, una pequeña editorial especializada en la literatura asiática, se destaca por su mayor cantidad de publicaciones en castellano de las obras de este escritor chino. De hecho, hasta abril de 2019, cuatro quintas partes de los libros de Mo Yan en España fueron publicadas por Kailas.

La cooperación entre Kailas y Mo Yan empezó en 2004. Al principio esta editorial tenía los derechos sobre seis títulos suyos hasta 2013, que después fueron renovados y ampliados al menos hasta 2020. Además, a través del nuevo contrato, Kailas se convirtió en el editor en exclusiva para el idioma español de las novelas del autor chino ( $M o$ Yan se queda en Kailas», El Mundo, 17.04.2019). Desde que fue fundada en 2004, ha publicado veintinueve traducciones del chino hasta abril de $2019^{5}$. Desde su fundación hasta 2011, las once obras de literatura china publicadas por Kailas son traducciones indirectas, realizadas a partir de versiones en lengua inglesa: cinco de Mo Yan y una, respectivamente, de Han Shaogong, Xu Xi, Wang Anyi, Li Cunxin, Guo Yue y Wu Xiaobo. Sin embargo, a partir de 2011, de las dieciocho traducciones del chino, las diez obras de Mo Yan se tradujeron directamente del chino, además de La oveja descarriada (2014), de Yu Dafu; Ciudad difunta (2018), de Jia Pingwa, y El viejo barco (2019), de Zhang Wei.

Se observa que no solo los textos, sino también los paratextos, se acercan a la lengua fuente. En cuatro de los quince libros de Mo Yan aparecen los títulos escritos en chino: Rana (蛙, 2011), El suplicio del aroma de sándalo (檀香刑, 2014), Trece pasos (十三步, 2015) y El manglar (红树

5. El dato proviene de la Base de datos de libros editados en España (consulta 24/06/2019). 
林, 2016), siendo destacable que la portada de esta última obra también contenga el nombre del autor escrito en chino "莫言》. La aparición de los caracteres chinos comenzó en 2011 con la primera traducción directa, Rana, y se extendió hasta 2016, con la única excepción de jBoom! (2013). Sin embargo, esa práctica no se ha vuelto a producir.

\section{CONClusión}

La traducción indirecta no solo hace más débil la conexión entre la literatura china y la española, sino que también hace que los lectores hispanohablantes inevitablemente reciban elementos del sistema de la lengua intermediaria sin saberlo. A lo largo del desarrollo de la traducción chino-española y la concesión del Premio Nobel de Literatura a Mo Yan, los círculos de traducción y edición prestan cada día más atención a tener un contacto más directo con la literatura china, como lo testimonian la mayor especialización de los traductores, un número mayor de traducciones directas de obras de Mo Yan y otros escritores chinos y los caracteres chinos aparecidos en los paratextos. Sin embargo, las traducciones chino-españolas todavía necesitan mejorar, ya que existen algunos errores en las versiones en español y es necesario retraducir las primeras traducciones indirectas.

\section{REFERENCIAS}

Ku, Menghsuan. 2010. «Reflexión de la traducción indirecta del chino al español. Ejemplo de la traducción de La vida y la muerte me está desgastandom. Confluenze 2 (1): 197-212.

MARíN LACARTA, Maialen. 2012. «Mediación, recepción y marginalidad: las traducciones de literatura china moderna y contemporánea en España». Tesis doctoral, Universitat Autònoma de Barcelona.

MENG Xiangchun. 2014. “葛浩文访谈” [Entrevista con Howard Goldblatt]. 中国翻译 [Traducción de China] 3: 72-77.

Mo, Yan. 2006. 生死疲劳 [La vida y la muerte me están desgastando]. Taipei: Cite.

Mo, Yan. 2008. Life and Death Are Wearing Me Out. Traducido por Howard Goldblatt. Nueva York: Arcade.

Mo, Yan. 2009. La vida y la muerte me están desgastando. Traducido por Carlos Ossés. Madrid: Kailas.

Mo, Yan. 2014. El suplicio del aroma de sándalo. Traducido por Blas Piñero Martínez. Madrid: Kailas.

ZHOU Chunxia. 2015. “莫言小说在西班牙的译介” [Traducción de obras de Mo Yan en España]. 南方文坛 / Southern Cultural Forum 3: 32-35. 